\title{
3D planar velocity measurements, using Mach-Zehnder interferometric-filter-based planar Doppler velocimetry (MZI-PDV) and imaging fibre bundles
}

\author{
Z. H. Lu, T. O. H. Charrett, H. D. Ford, R. P. Tatam \\ Engineering Photonics Group, Centre for Photonics and Optical Engineering, School of Engineering, \\ Cranfield University, Cranfield, Bedford, MK43 OAL, UK.
}

\begin{abstract}
Three component planar flow-field measurements are made using imaging fibre bundles to port different views of the measurement plane, defined by a laser light sheet, to a single imaging head. The Doppler frequency shifts of light scattered by particles entrained in the flow are transduced to intensity variations using a Mach-Zehnder interferometric filter. The free spectral range of the filter can be selected by adjusting the optical path difference of the interferometer. This allows the velocity measurement range, sensitivity and resolution to be varied. Any laser wavelength may be used. A phase locking system has been designed to stabilise the interferometric filter.
\end{abstract}

Keywords: Planar Doppler velocimetry, PDV, Mach-Zehnder interferometer, flow measurement, phase control

\section{INTRODUCTION}

Knowledge of fluid velocities is essential in a variety of engineering applications, e.g. wind tunnel measurements for vehicle prototypes in the aerospace and automotive industries, and internal flows in, for example, turbomachinery. To build up a $3 \mathrm{D}$ velocity vector map with good spatial resolution over an extended region requires a large number of individual measurements and can be very time consuming. Planar techniques, however, provide velocity information over a two-dimensional region with a single measurement. Planar Doppler velocimetry (PDV) $)^{1,2}$ is a Doppler-based planar technique measuring optical frequency shifts of laser light scattered by moving particles or molecules in a flow, and yielding the flow velocity, using the Doppler equation.

$$
\Delta v=\frac{v_{L}(\hat{o}-\hat{i}) \cdot V}{c}
$$

where $\Delta v=v-v_{L}$ is the difference between the scattered light frequency, $v$, and the original laser light frequency, $v_{L} . V$ is the velocity vector associated with the scattering particles and $c$ the free space speed of light. $\hat{o}$ and $\hat{\imath}$ are unit vectors in the observation and illumination directions, respectively (Fig. 1). In conventional PDV, an atomic or molecular vapour cell, often containing iodine cell, is used to transduce the Doppler shift in the scattered laser light to an intensity variation. By selecting the illumination frequency to coincide with an absorption line of the gas, the received intensity at the CCD camera is directly related to the Doppler shift, and hence the flow velocity. A reference camera, viewing the flow directly, is needed to normalise the signal for variations in scattered light intensity due to effects other than the flow velocity. However, the use of molecular gas has several disadvantages. The first is that the choice of lasers is limited by the requirement to tune to an appropriate absorption line. The second is that the transfer function is determined by the form of the gas absorption line and therefore the sensitivity can not be increased, although it is possible to broaden the spectral feature, thus reducing the sensitivity whilst increasing the range, by the addition of buffer gases ${ }^{3}$.

Optical interferometry, another technique for frequency-to-intensity conversion, also allows the visualisation of a flow field. The Doppler-shifted light scattered from a flow leads to a change of the light intensity distribution in an interference pattern which relates directly to the flow velocity. Recently, a Mach-Zehnder interferometric filter based planar Doppler velocimetry (MZI-PDV) ${ }^{4}$ technique has been developed using a MZI filter instead of a molecular filter as the frequency-to-intensity converter. The use of a MZI provides two complementary outputs, allowing the scattered light intensity to be normalised by taking the difference of the two outputs divided by the sum. The path length imbalance can be selected to correspond to the expected range of velocities in a given flow situation. Any continuous wave or pulsed

19th International Conference on Optical Fibre Sensors, edited by David Sampson, Stephen Collins, Kyunghwan Oh, Ryozo Yamauchi, Proc. of SPIE Vol. 7004, 70040I, (2008) 0277-786X/08/\$18 doi: 10.1117/12.786544 
laser of sufficient power can be used as the light source. A typical PDV system gives only the component of the flow velocity in the $(\hat{o}-\hat{\imath})$ direction with a magnitude of $|(\hat{o}-\hat{\imath}) \cdot V| / \lambda$. To obtain all three components of the velocity, an imaging fibre bundle $e^{5,6}$ has been used to measure three component velocities simultaneously by porting multiple views to a single detector head. Here, the MZI-PDV technique was modified using an imaging fibre bundle to allow the measurement of multiple velocity components.

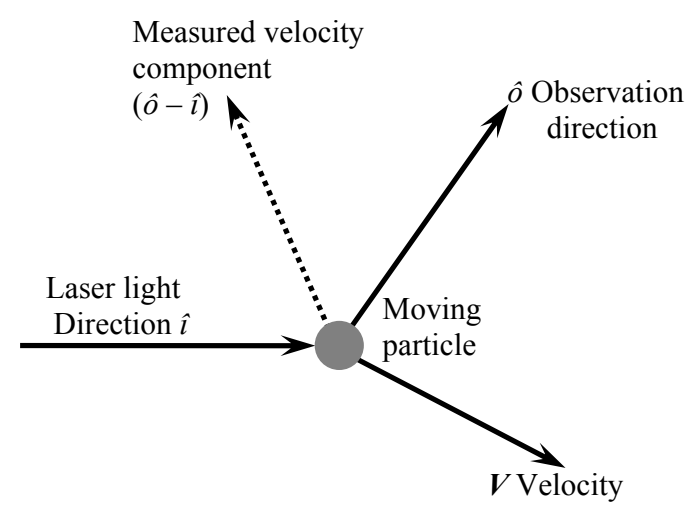

Fig. 1. Diagram of illumination and observation directions for light scattering from a moving particle.

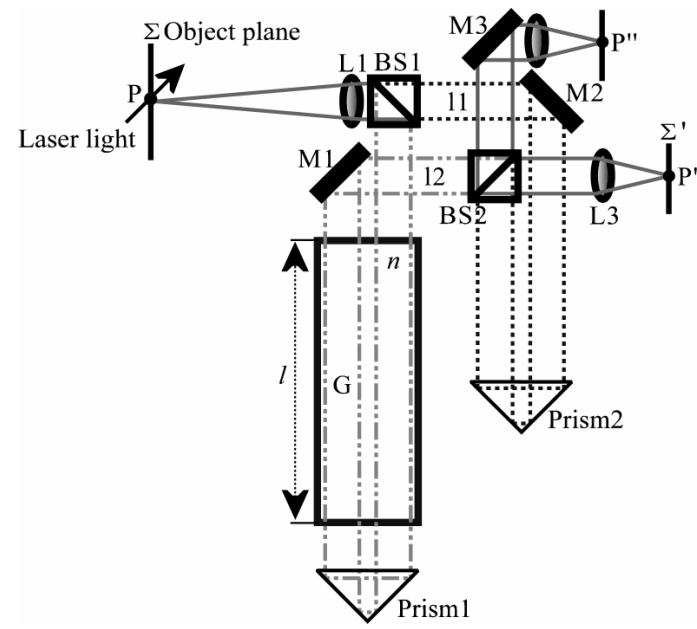

Fig. 2. An unbalanced MZI for PDV. The beam paths are shown in different styles: path 11 is dashed, 12 is dot-dashed and the combined path is solid.

\section{MACH-ZEHNDER PDV INTERFEROMETER (MZI-PDV)}

Fig. 2 shows the arrangement of an unbalanced Mach-Zehnder PDV interferometer (MZI-PDV). A laser light sheet, of frequency $v_{L}$, illuminates the object plane $\Sigma$ located in the flow. Light scattered from particles seeded in the flow is collected and coupled into the interferometer. Frequency variations caused by the Doppler shift are converted into variations in the recorded intensities on CCD detectors located at $\Sigma^{\prime}$, and $\Sigma^{\prime \prime}$. The scattered light coming from the object plane $\Sigma$ is collimated by lens L1 and then divided by BS1 (beam splitter, 50:50 split ratio) into two beams of equal intensity, which follow paths 11 and 12. The light in path 11 is turned at mirror M2 and prism 2 before being split by BS2 and imaged onto the two detectors located at $\Sigma$ ', and $\Sigma^{\prime \prime}$ using lenses L2 and L3. The second path, 12 passes through a glass block $G$, with a length $l$ and refractive index $n$. The light is retroreflected by prism 1, making a second pass through the glass block. It is then turned at mirror, M1 and recombined with the light in the first path at BS2. For a MZI, the normalised intensity $I_{N}$ can be found as

$$
I_{N}=\frac{I_{1}-I_{2}}{I_{1}+I_{2}} \propto V_{i} \cdot \cos \left(\frac{2 \pi}{c} \Delta l \cdot v\right)
$$

where $I_{1}, I_{2}$ are intensities at the two outputs of the MZI, $\Delta l$ is the optical path difference between the two arms, and $V_{i}$ is the interference fringe visibility determined by the transmission performance of the whole optical set-up. The normalised intensity, $I_{N}$, is a function of only the optical path difference $\Delta l$, the light source frequency $v$, and is independent of the original input intensity $\left(I_{1}+I_{2}\right)$. Therefore, any Doppler shift of the scattered light will cause a variation of the normalised intensity providing a fixed optical path difference.

The optical path difference can be varied by selecting the length $l$ of the glass block to correspond to the expected measurement range. This ability is much more limited in conventional PDV systems that use molecular filters. The primary purpose of the glass block is to correct for the difference in magnification and focal distance in the two arms of the interferometer.

\section{EXPERIMENTAL SETUP}

A schematic of the experimental arrangement used in this work is shown in Fig. 3. The illumination laser was a continuous wave argon ion laser, allowing time averaged velocity measurements to be made. Light from the laser was 
coupled into an optical fibre through a Faraday isolator, and the output was formed into a light sheet using a prismscanning device that scanned the beam rapidly across the measurement area. The scattered light from a flow region was imaged using standard SLR camera lenses and ported to the MZI using a coherent array of fibres that is split into four channels. Each channel comprises $600 \times 500$ fibres, $8 \mu \mathrm{m}$ in diameter and positioned on $10 \mu \mathrm{m}$ centres. The MZI was constructed using an infinity-corrected microscope optical system consisting of a matched objective and a tube lens. The two complementary output images of the MZI were captured by the two CCD cameras. In order to prevent the interferometer phase drift due to temperature variations and mechanical vibrations, a phase locking system was designed using a feedback loop controller to adjust the path difference. This uses a $633 \mathrm{~nm}$ beam from a single-frequency stabilised He-Ne laser, together with green blocking filters, to provide signals for a custom designed PID electronic circuit.

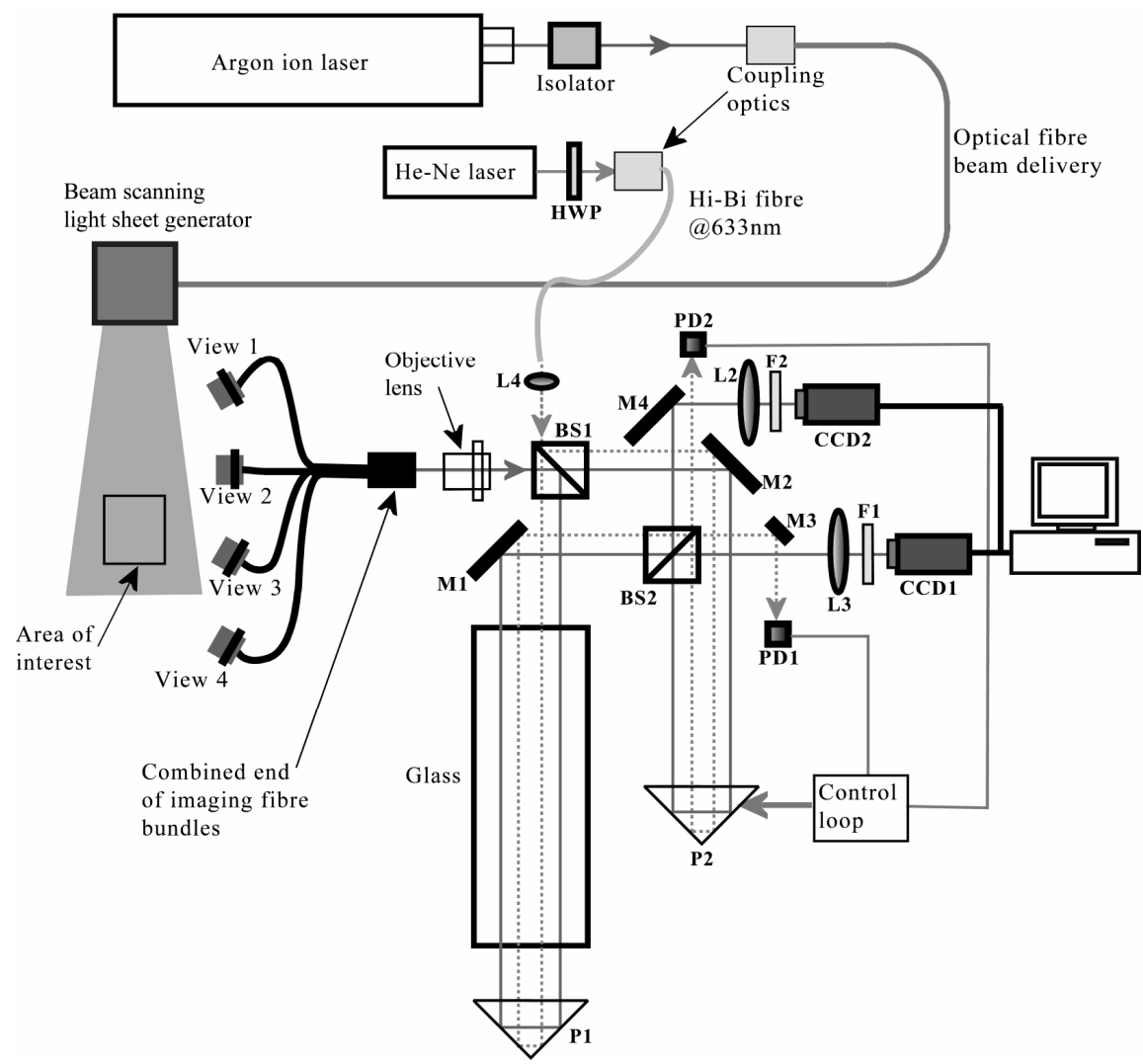

Fig. 3. The experimental arrangement; including detail of the phase locking system to stabilise the MZI.

HWP: half-wave plate; BS1, 2: non-polarisation beam splitters; L2, 3: tube lenses; L4: coupling lens; M1, 2, 3, 4: mirrors; F1, 2: green filters; P1, 2: right-angle prisms. Beam path for measurement system shown as solid line, beam path for phase locking system shown as a dotted line.

\section{RESULTS}

The system was initially tested as a single velocity component system, in which the imaging fibre bundle was replaced by a camera lens, giving a single viewing direction. This was calibrated using measurements on a rotating disc, which provides a well-known velocity field. It was then used to make measurements on a seeded air jet, with a $20 \mathrm{~mm}$ diameter nozzle. The exit velocity of the jet is $\sim 85 \mathrm{~ms}^{-1}$. The sensitivity vector is at an angle of $\sim 45^{\circ}$ from the main flow direction determined from measurements of the observation and illumination positions. Fig. 4 shows the measured velocity component of the air jet flow. The velocity follows the expected form for a jet, and measured velocities correspond closely to values calculated using a simple empirical equation. The system was then extended to measure three velocity components, using the imaging fibre bundle as the input into the MZI. The rotating disc was again used to validate the system. The rotating disc was $200 \mathrm{~mm}$ in diameter giving a maximum velocity in the field of view of $\sim 30 \mathrm{~ms}^{-1}$ at the disc edge, as measured using an optical tachometer. Data from each of the observation directions was processed to yield a velocity component, with the direction given by $(\hat{o}-\hat{\imath})$. These measured velocity components were then converted to orthogonal components and a smoothing filter was applied. Fig. 5(a)-(c) show examples of these computed orthogonal 
components. The calculated orthogonal velocity components show the expected behaviour; horizontal velocity varies linearly across the height of the disc, and vertical velocity across the width. The out-of-plane component is, as would be expected, close to zero. Values agree well with those calculated from the optical tachometer measurement. The velocity standard deviation is about $\pm 2 \mathrm{~ms}^{-1}$ for the images shown.

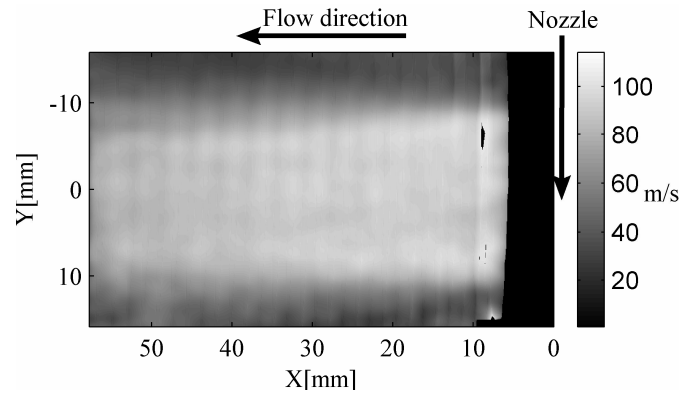

Fig. 4. The measured velocity component of the air jet flow made using the MZI-PDV system.

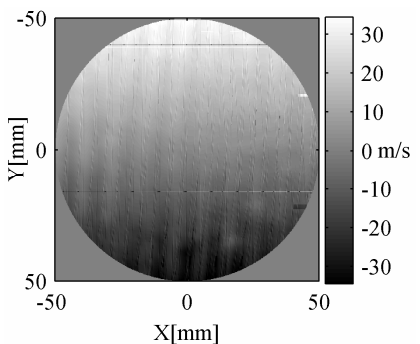

(a)

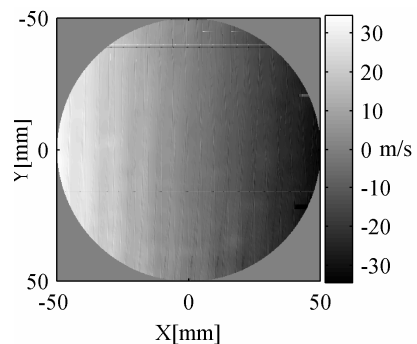

(b)

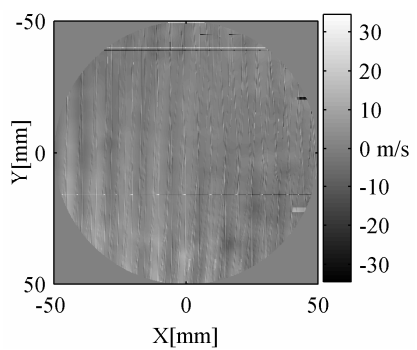

(c)

Fig. 5. Velocity fields from a rotating disc. (a) Typical computed horizontal (U) velocity component. (b) Typical computed vertical (V) velocity component. (c) Typical computed out-of-plane (W) velocity component.

\section{CONCLUSIONS}

A PDV technique using a MZI filter and an imaging fibre bundle has been demonstrated that is capable of measuring all three components of velocity, across a plane defined by a light sheet. The path length imbalance of the interferometer can be selected to correspond to the expected range of velocities in a given flow situation. Any wavelength may be used as the light source. The use of the imaging bundle also allows greater flexibility in the experimental arrangement.

\section{ACKNOWLEDGMENTS}

Zenghai Lu acknowledges an Overseas Research Scholarship (ORS) award from the Committee of Vice Chancellors and Principals, UK. This work was supported by a Paul Instrument Fund grant from the Royal Society, UK.

\section{REFERENCES}

1. H. Komine, S. Brosnam, A. Littion, and E. Staepperts, "Real-time Doppler Global Velocimetry", AIAA 29th Aerospace Sciences Meetings, Reno, Nevada, Paper 91-0337, (1991).

2. G. S. Elliott, and T. J. Beutner, "Molecular filter based planar Doppler velocimetry", Progress in Aerospace Sciences, 35(8), 799-845 (1999).

3. V. S. S. Chan, A. L. Heyes, D. I. Robinson, and J. T. Turner, "Iodine absorption filters for Doppler global velocimetry", Meas. Sci. Technol., 6(6), 784-794 (1995).

4. Z. H. Lu, H. D. Ford, T. O. H. Charrett and R. P. Tatam, "Mach-Zehnder interferometric filter based planar Doppler velocimetry", J. Opt. A: Pure Appl. Opt., 9, 1002-1013 (2007).

5. D. S. Nobes, H. D. Ford and R. P. Tatam, "Instantaneous, three-component planar Doppler velocimetry using imaging fibre bundles", Experiments in Fluids, 36(1), 3-10 (2004).

6. T. O. H. Charrett, H. D. Ford and R. P. Tatam, "Single camera 3D planar Doppler velocity measurements, using two frequency Planar Doppler Velocimetry (2v-PDV) and imaging fibre bundles", 44th AIAA Aerospace Sciences Meeting and Exhibit, Reno, Nevada, AIAA-2006-47 (2006). 